\title{
Rotor-Bearing System Stability Performance Comparing Hybrid versus Conventional Bearings
}

\author{
Julio Gomez-Mancilla \\ Laboratorio de Vibraciones \& Rotodinamica ESIME, Instituto Politecnio Nacional, Edificio 5, 3er Piso, Colonia Zacatenco, \\ 07300 Mexico, Distrito Federal, Mexico \\ Email:jcgomez@ipn.mx

\section{Valeri Nosov} \\ Laboratorio de Vibraciones \& Rotodinamica ESIME, Instituto Politecnio Nacional, Edificio 5, 3er Piso, Colonia Zacatenco, \\ 07300 Mexico, Distrito Federal, Mexico \\ Email:vnossov@ipn.mx
}

\section{Gerardo Silva-Navarro}

Seccion de Mecatronica, Centro de Investigacion y de Estudios Avanzados del IPN, A.P. 14-740, 07360 Mexico, Distrito Federal, Mexico

Email: gsilva@cinvestav.mx

Received 1 May 2004

\begin{abstract}
New closed-form expressions for calculating the linear stability thresholds for rigid and flexible Jeffcott systems and the imbalance response for a rotor supported on a hybrid bearing are presented. For typical bearings characteristics, expressions yield stability thresholds practically equal to those reported by Lund (1966). The hybrid bearing design has a single injection port whose location is so chosen to stabilize the bearing performance and to reduce the steady equilibrium attitude angle. Rotordynamics coefficients graphs for conventional and pressurized bearings, as functions of bearing equilibrium eccentricity and/or Sommerfeld number, are presented. Using the rotordynamics coefficients into the expressions for the corresponding velocity thresholds and the imbalance response, the system stability and vibration performances are estimated and analyzed. When comparing the Jeffcott flexible shaft supported on two journal bearings of the conventional type with the hybrid type, the results show a clear superiority of the pressurized design as far as stability behavior is concerned. Specifically for cases of flexible shafts with similar characteristics to those used in industry, the analysis shows that this design yields velocity thresholds $25 \%-40 \%$ higher compared to the conventional circular ones. Also this bearing displays nonlinear feeding pressure behavior, and it is capable of reducing the synchronous vibration amplitude in most speed ranges, except around the critical speed; moreover, for certain Jeffcott configurations the amplitude reduction can be substantial.
\end{abstract}

Keywords and phrases: Jeffcott rotor, hybrid bearing, imbalance response, pressurized rotordynamics coefficients, stability threshold.

\section{INTRODUCTION}

By hybrid we mean a circular journal bearing that works with both, a hydrodynamic wedge plus a lubricant injected at substantial pressure. Bently et al. [1,2] proposed a hybrid bearing design capable of removing the oilwhirl bearing instabilities. As presented by Kucherenko and GomezMancilla [3], turbomachinery seals located near the midspan

This is an open access article distributed under the Creative Commons Attribution License, which permits unrestricted use, distribution, and reproduction in any medium, provided the original work is properly cited. exert a great dynamic influence in spite of their small values. In [3] the equations of motion using dimensionless parameters are developed, allowing us to notice how small changes on the stiffness largely affect the stability properties. Oilwhirl is a milder instability problem in comparison to oilwhip and steamwhirl, all occurring at subsynchronous vibration frequencies. In general a moderate nonlinear bearing force is enough to control growth of oilwhirl vibration amplitudes, but this is not so for the other two instabilities. On the other hand, acting at a susceptible location by producing a relatively small change in midspan effective stiffness Bently et al. [2] has put up a rotor-bearing-seal system capable 
of postponing the oilwhip instability. Therefore, location of the active control element is of fundamental importance to achieve high dissipating efficiency, that is, the squeeze film dampers are conveniently located at strategic points. Although bearing locations near the rotor ends are not optimal, here shaft ends are bearing locations used to estimate system stability performance.

Recently some interesting bearing design capable of changing effectively the stability and behavior of supported rotors have been proposed by Bently et al. [1, 2], GomezMancilla [4], Dimofte and Hendricks [5], just to mention three works. The rotor-bearing stability is considerably influenced by the fluid film, modeled by the rotordynamics coefficients, which in turn are functions of machine operation speed, lubricant viscosity temperature, and so forth. In the Laboratorio de Vibraciones \& Rotodinamica ESIME, ESIME$I P N$, it has been noted that rotor-bearing instabilities can be controlled and the operational stable range and the vibration performance can be substantially improved by incrementing the lubricant pressure supply in a bearing. Therefore, adequate changes of the rotordynamics coefficients, caused by the lubricant pressure supply increment, will favorably postpone the stability threshold of a machine, sometimes in a considerable manner (Ordoñez-Pantoja [6]). Briefly it can be mentioned that there exist other bearing designs such as the tilting pad, the multilobe, and elliptic bearings, all of which are intrinsically more stable when compared to the circular one studied here. See, for instance, [7] for stability performances of circular, four-lobe, and tilting pad bearings. Nevertheless this study does not only analyze stability issues but also compares rotor-bearing synchronous vibration responses, where this hybrid design performs quite well.

In this paper, a formula for the calculus of the stability threshold, which is obtained through the Liénard-Chipart criterion is used (Antonio-Garcia et al. [8] ; Afanasiev et al. [9]; Demidovich [10]). The geometric configuration of the bearing shown in Figure 1a provides the same benefits as the conventional lubricant pressure supply required to achieve the classical supply lubrication. For the infinitely rigid shaft, the hybrid bearing leads to a very high increase in the speed threshold and a significant but moderate improvement as the shaft flexibility increases. Hence the advantage of modifying the bearing can be seen in improving the stability threshold.

\section{ROTORDYNAMICS COEFFICIENTS IN CASE OF LUBRICANT INJECTION}

The physical configuration is shown in Figure 1a, where the lubricant injection port is located on the upper top of the bearing. The purpose of this design is to exert a pressure downward force especially useful at high Sommerfeld numbers, when the attitude angles are particularly large. There is no danger in reducing the minimum oil film since a circular bearing is generally more unstable at small eccentricities and large attitude angles, a situation which is corrected by the injection pressure force.

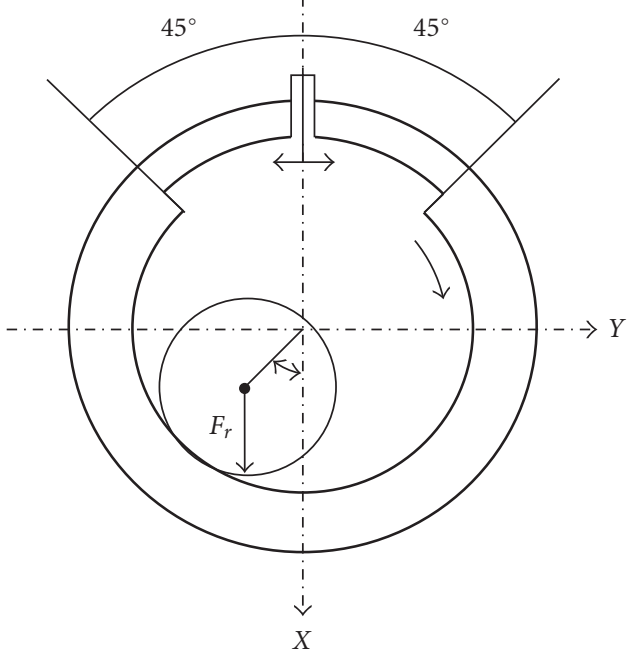

(a)

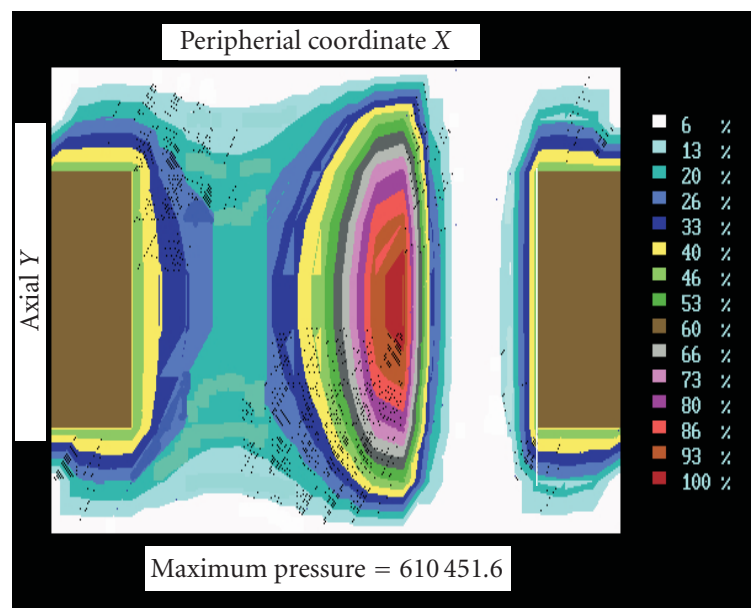

(b)

FIGURE 1: The analyzed bearing: (a) physical description and geometrical configuration and (b) typical pressure field used to compute coefficients; notice the feed port pressurized area at $P_{s}=10$.

The rotordynamics coefficients $\left(k_{i j}, c_{i j}\right)$ for Figure la configuration are computed using the CHUMA software developed by Gomez-Mancilla and Dimarogonas [11] for fixed multipad journal bearings with or without preload. CHUMA solves numerically the Reynolds equation, estimating the equilibrium point and corresponding pressure field using the Stieber-Swift boundary conditions, then computes bearing reaction forces and expanding them into Taylor series obtains linear and nonlinear bearing stiffness and damping coefficients. An incompressible lubricant and a groove depth significant enough to maintain a constant pressure zone in the port area is assumed as illustrated in Figure 1b.

The dimensionless increase of bearing pressure above classical supply is expressed by

$$
P_{s}=\frac{p_{s}}{P}
$$


where $p_{s}$ denotes the pressure supply due to additional lubricant injection in the bearings and $P=F_{0} / L D$ is the bearing static pressure load, where $F_{0}$ is the bearing static load. Typical pressure supply values $P_{s}$ are 0 (classical supply without additional pressurization), 3,6 , and 10 . The value $p_{s}=0 P$ refers to the case of maintaining a sufficient lubricant supply to keep a constant fluid film between the journal and the bearing.

Program outputs include the stiffness $k_{i j}$ and damping $c_{i j}$ coefficients in the dimensionless form, which are defined as follows:

$$
\tilde{k}_{i j}=\frac{C_{r}}{F_{0}} k_{i j}, \quad \tilde{c}_{i j}=\frac{\omega C_{r}}{F_{0}} c_{i j}
$$

where $C_{r}$ denotes the bearing radial clearance and $\omega$ the operation angular speed.

Figures 2 and 3 illustrate different behavior for the rotordynamics coefficients presented as functions of the Sommerfeld number and using the coordinates $(x, y)$ in Figure 1a. Due to the existence of a negative portion in the $k_{x y}$ curve (printed in dashed line), absolute values for this coefficient are plotted. In Figure 3a it is important to notice that the $k_{x y}$ axis crossing, from negative to positive values, occurs at significantly larger Sommerfeld numbers, a situation which can be interpreted as beneficial for bearing stability.

\section{STABILITY THRESHOLD FOR RIGID ROTORS}

The Jeffcott model equations describe the motion of a symmetrical rotor-bearing system with a $2 m$ disc mass located in the middle of the shaft and supported on two hydrodynamic bearings on each rotor end. The shaft can be rigid or flexible. The Jeffcott rotor equations can be presented in dimensionless form as follows:

$$
p^{2}\left\{\begin{array}{c}
\bar{X}^{\prime \prime} \\
\bar{Y}^{\prime \prime}
\end{array}\right\}+\left[\tilde{C}_{i j}\right]\left\{\begin{array}{c}
\bar{X}^{\prime} \\
\bar{Y}^{\prime}
\end{array}\right\}+\left[\tilde{K}_{i j}\right]\left\{\begin{array}{l}
\bar{X} \\
\bar{Y}
\end{array}\right\}=\frac{p^{2} a}{C_{r}}\left\{\begin{array}{l}
\cos \tau \\
\sin \tau
\end{array}\right\},
$$

where $p^{2}=C_{r} m \omega^{2} / F_{0}, \bar{X}=X / C_{r}, \bar{Y}=Y / C_{r}, \omega$ is the angular speed, $C_{r}$ is the bearing radial clearance, and $a$ is the disc residual imbalance. The variables with quoted marks denote derivatives with respect to the dimensionless time $\tau=\omega t$. The corresponding characteristic equation for the system (3) is given by the fourth-order polynomial

$$
P_{4}(\lambda)=\lambda^{4}+\frac{\Sigma(c)}{p^{2}} \lambda^{3}+\frac{p^{2} \Sigma(k)+\Delta(c)}{p^{4}} \lambda^{2}+\frac{\Delta(c, k)}{p^{4}} \lambda+\frac{\Delta(k)}{p^{4}},
$$

where

$$
\begin{gathered}
\Sigma(c)=\tilde{c}_{x x}+\tilde{c}_{y y}, \quad \Sigma(k)=\tilde{k}_{x x}+\tilde{k}_{y y}, \\
\Delta(c)=\tilde{c}_{x x} \tilde{c}_{y y}-\tilde{c}_{y x} \tilde{c}_{x y}, \\
\Delta(k)=\tilde{k}_{x x} \tilde{k}_{y y}-\tilde{k}_{y x} \tilde{k}_{x y}, \\
\Delta(c, k)=\tilde{c}_{x x} \tilde{k}_{y y}+\tilde{c}_{y y} \tilde{k}_{x x}-\tilde{c}_{x y} \tilde{k}_{y x}-\tilde{c}_{y x} \tilde{k}_{x y} .
\end{gathered}
$$

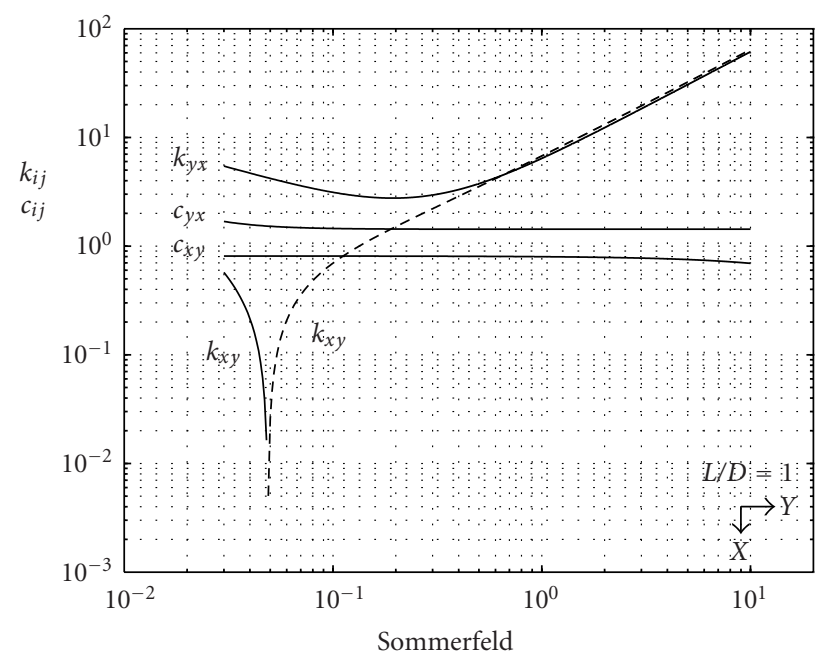

(a)

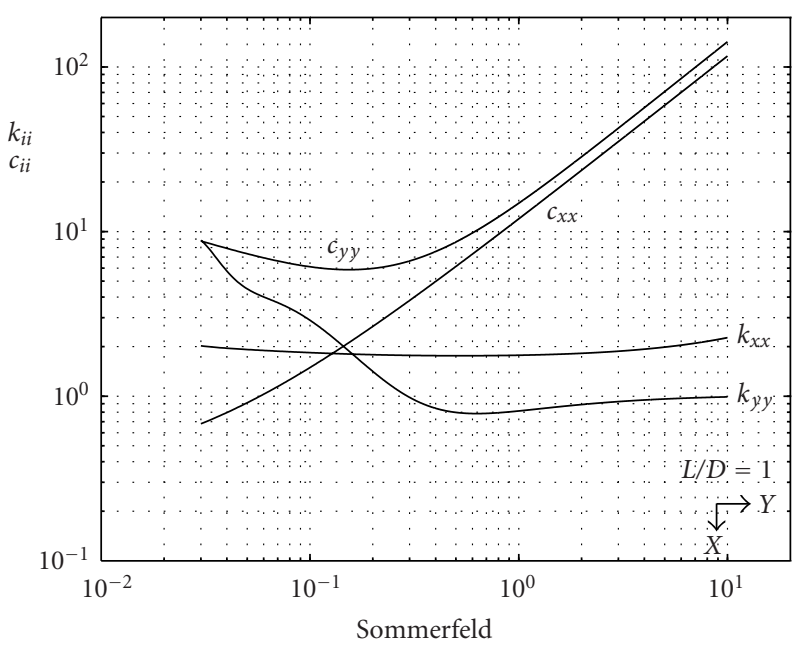

(b)

FIGURE 2: Stiffness and damping rotordynamics coefficients of cylindrical bearing for $P_{s}=0$, equivalent to conventional circular journal bearings: (a) cross-coupled coefficients and (b) direct coefficients.

The characteristic polynomial (4) can be rewritten as

$$
P_{4}(\lambda)=\lambda^{4}+b_{1} \lambda^{3}+b_{2} \lambda^{2}+b_{3} \lambda+b_{4},
$$

where

$$
\begin{gathered}
b_{1}=\frac{\Sigma(c)}{p^{2}}, \quad b_{2}=\frac{p^{2} \Sigma(k)+\Delta(c)}{p^{4}}, \\
b_{3}=\frac{\Delta(c, k)}{p^{4}}, \quad b_{4}=\frac{\Delta(k)}{p^{4}} .
\end{gathered}
$$

The necessary and sufficient conditions for stability are obtained from the Liénard-Chipart criterion (Afanasiev et al. [9]; Demidovich [10]). In particular, for the characteristic 


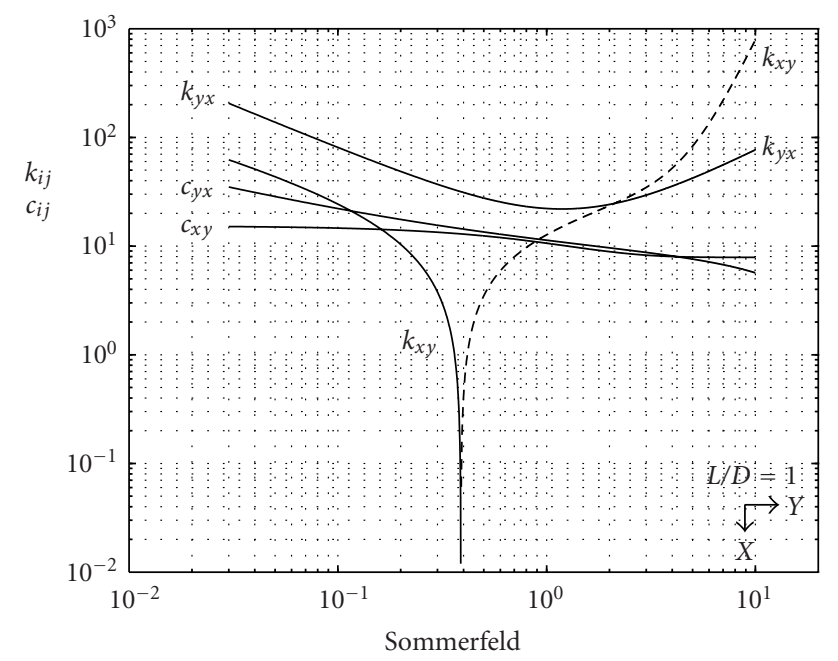

(a)

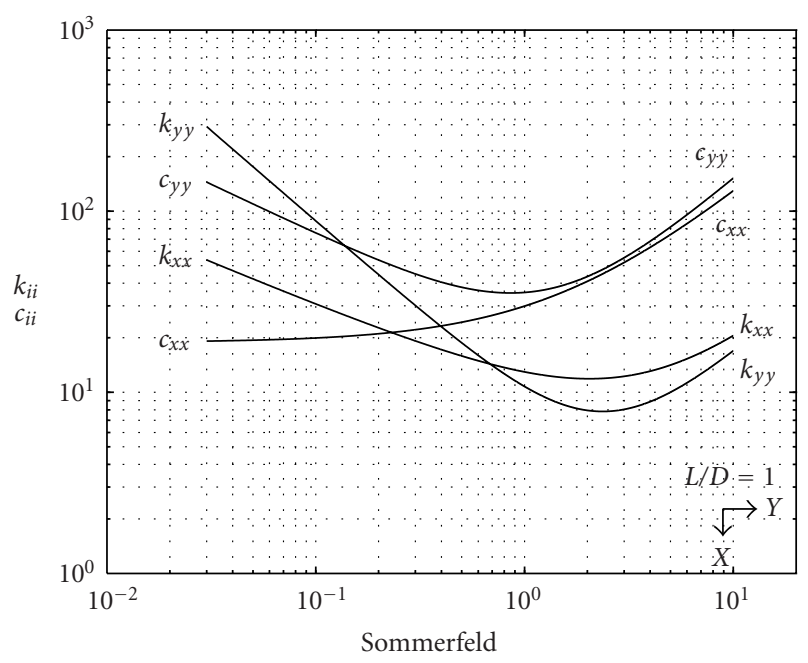

(b)

Figure 3: Stiffness and damping rotordynamics coefficients of hybrid bearing design for $P_{s}=10$ : (a) cross-coupled coefficients and (b) direct coefficients.

polynomial $P_{4}(\lambda)$ in (6) the necessary and sufficient conditions for stability are that all its coefficients $b_{i}>0, i=1, \ldots, 4$ and

$$
\Delta_{1}=\left|b_{1}\right|>0, \quad \Delta_{3}=\left|\begin{array}{ccc}
b_{1} & 1 & 0 \\
b_{3} & b_{2} & b_{1} \\
0 & b_{4} & b_{3}
\end{array}\right|>0
$$

where $|\cdot|$ denotes the determinant function. The square of the stability threshold $p_{\mathrm{RS}, \text { thr }}^{2}$ for a rigid shaft configuration, obtained through the Lienard-Chipart criterion (8), is obtained as

$$
p_{\mathrm{RS}, \mathrm{thr}}^{2}=\frac{\Delta(c) \Delta(c, k) \Sigma(c)}{\Delta^{2}(c, k)+\Delta(k) \Sigma^{2}(c)-\Sigma(k) \Delta(c, k) \Sigma(c)} .
$$

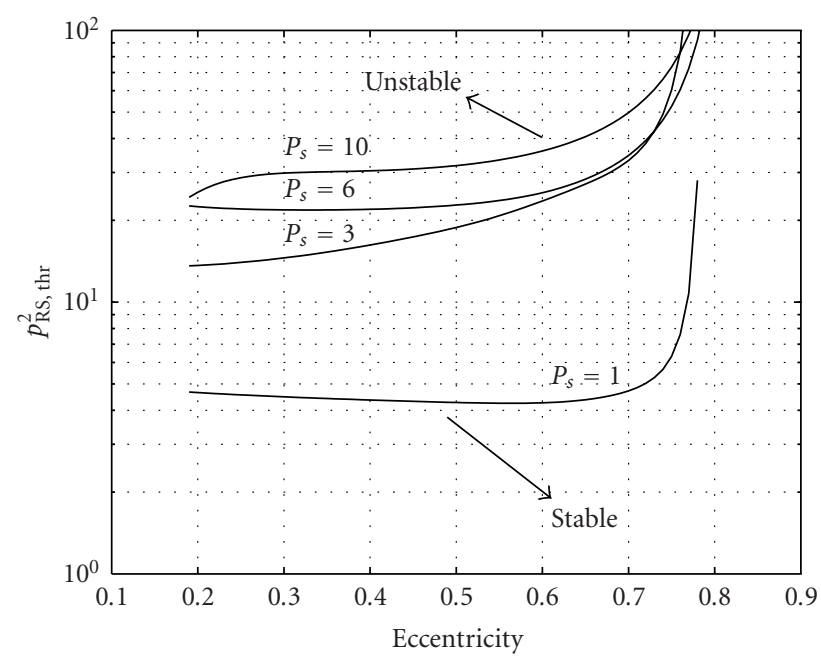

(a)

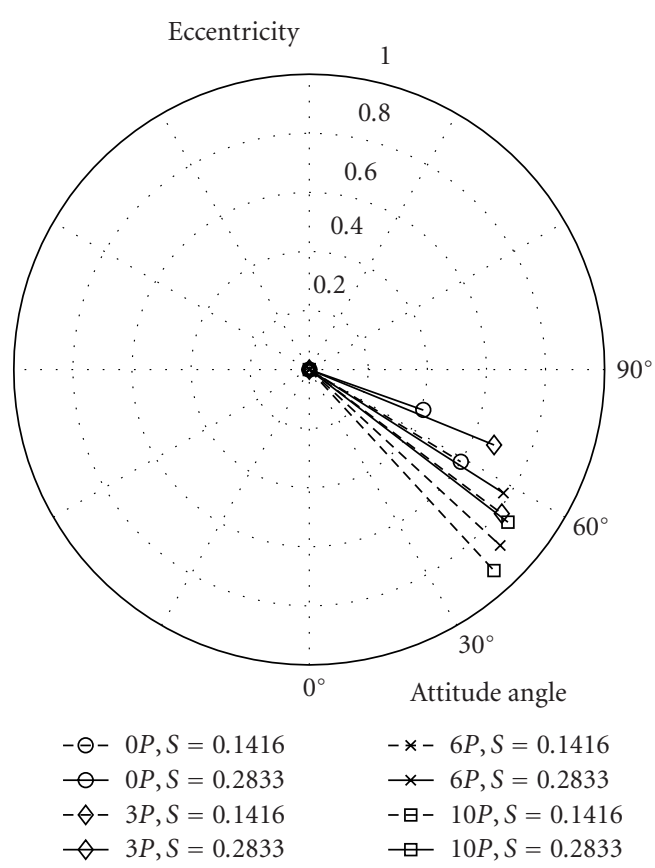

(b)

FIGURE 4: Rigid shaft stability behavior for conventional and hybrid designs: (a) $p_{\mathrm{RS}, \text { thr }}^{2}$ for different lubricant pressure supplies and (b) equilibrium locus for $S=0.1416,0.2833$ where eccentricities and attitude angles for four injection pressures are indicated.

Figure 4a illustrates a very significant improvement on the threshold as the feed pressure is increased from $P_{s}=0$ to $P_{s}=10$, nearly one order of magnitude for $p_{\mathrm{RS}, \text { thr }}^{2}$. In addition, Figure $4 \mathrm{~b}$ clearly indicates that increasing of injection pressure effectively reduces the equilibrium attitude angle, estimated at two different Sommerfeld numbers, a feature characterizing stability. 


\section{STABILITY THRESHOLD FOR FLEXIBLE ROTORS}

The Jeffcott model with shaft flexibility and mass imbalance at the disc is obtained as

$$
\begin{gathered}
m \ddot{x}+k\left(x-x_{0}\right)=m a_{x} \omega^{2} \cos \omega t-m a_{y} \omega^{2} \sin \omega t, \\
m \ddot{y}+k\left(y-y_{0}\right)=m a_{x} \omega^{2} \sin \omega t+m a_{y} \omega^{2} \cos \omega t, \\
\tilde{k}_{x x} x_{0}+\tilde{k}_{x y} y_{0}+\tilde{c}_{x x} \dot{x}_{0}+\tilde{c}_{x y} \dot{y}_{0}=k\left(x-x_{0}\right), \\
\tilde{k}_{y x} x_{0}+\tilde{k}_{y y} y_{0}+\tilde{c}_{y x} \dot{x}_{0}+\tilde{c}_{y y} \dot{y}_{0}=k\left(y-y_{0}\right),
\end{gathered}
$$

where the coordinates $(x, y)$ and $\left(x_{0}, y_{0}\right)$ are the disc center mass and the bearing center mass displacements, respectively; $k=K / 2$, with $K$ describing the shaft stiffness; $a_{x}, a_{y}$ are components of the disc mass imbalance, and $\omega$ is shaft angular speed. Equations (10)-(11) in dimensionless coordinates are rewritten as

$$
\begin{array}{r}
p^{2} \bar{x}^{\prime \prime}+\frac{1}{\gamma}\left(\bar{x}-\bar{x}_{0}\right)=p^{2}\left[\left(\frac{a_{x}}{c_{r}}\right) \cos \tau-\left(\frac{a_{y}}{c_{r}}\right) \sin \tau\right], \\
p^{2} \bar{y}^{\prime \prime}+\frac{1}{\gamma}\left(\bar{y}-\bar{y}_{0}\right)=p^{2}\left[\left(\frac{a_{x}}{c_{r}}\right) \sin \tau+\left(\frac{a_{y}}{c_{r}}\right) \cos \tau\right], \\
\tilde{k}_{x x} \bar{x}_{0}+\tilde{k}_{x y} \bar{y}_{0}+\tilde{c}_{x x} \bar{x}_{0}^{\prime}+\tilde{c}_{x y} \bar{y}_{0}^{\prime}=\frac{1}{\gamma}\left(\bar{x}-\bar{x}_{0}\right), \\
\tilde{k}_{y x} \bar{x}_{0}+\tilde{k}_{y y} \bar{y}_{0}+\tilde{c}_{y x} \bar{x}_{0}^{\prime}+\tilde{c}_{y y} \bar{y}_{0}^{\prime}=\frac{1}{\gamma}\left(\bar{y}-\bar{y}_{0}\right),
\end{array}
$$

where $\bar{x}=x / C_{r}, \bar{y}=y / C_{r}, \bar{x}_{0}=x_{0} / C_{r}$, and $\bar{y}_{0}=y_{0} / C_{r}$ are dimensionless displacements, $\gamma=\delta / C_{r}$ is the dimensionless static deflection with respect to a shaft midspan static deflection $\delta=F_{0} / k$. The characteristic equation of the system (12)-(13) is the sixth-order polynomial

$$
\begin{aligned}
& \lambda^{6} \gamma^{2} \Delta(c)+\lambda^{5}\left[\gamma \Sigma(c)+\gamma^{2} \Delta(c, k)\right] \\
& +\lambda^{4}\left[1+\gamma^{2} \Delta(k)+\gamma \Sigma(k)+2 \gamma \frac{\Delta(c)}{p^{2}}\right] \\
& +\lambda^{3}\left[\frac{\sum(c)}{p^{2}}+2 \gamma \frac{\Delta(c, k)}{p^{2}}\right] \\
& +\lambda^{2}\left[\frac{\sum(k)}{p^{2}}+\frac{\Delta(c)}{p^{4}}+2 \gamma \frac{\Delta(k)}{p^{2}}\right] \\
& +\lambda \frac{\Delta(c, k)}{p^{4}}+\frac{\Delta(k)}{p^{4}}=0,
\end{aligned}
$$

where notations (5) are used. This polynomial can be expressed as

$$
P_{6}(\lambda)=b_{0} \lambda^{6}+b_{1} \lambda^{5}+b_{2} \lambda^{4}+b_{3} \lambda^{3}+b_{4} \lambda^{2}+b_{5} \lambda+b_{6},
$$

where

$$
\begin{gathered}
b_{0}=\gamma^{2} \Delta(c), \quad b_{1}=\gamma \Sigma(c)+\gamma^{2} \Delta(c, k), \\
b_{2}=1+\gamma^{2} \Delta(k)+\gamma \Sigma(k)+2 \gamma \frac{\Delta(c)}{p^{2}}, \\
b_{3}=\frac{\Sigma(c)}{p^{2}}+2 \gamma \frac{\Delta(c, k)}{p^{2}}, \quad b_{4}=\frac{\Sigma(k)}{p^{2}}+\frac{\Delta(c)}{p^{4}}+2 \gamma \frac{\Delta(k)}{p^{2}}, \\
b_{5}=\frac{\Delta(c, k)}{p^{4}}, \quad b_{6}=\frac{\Delta(k)}{p^{4}} .
\end{gathered}
$$

Application of the Liénard-Chipart criterion to the characteristic polynomial (15) leads to the necessary and sufficient conditions for stability of the system, that is, all its coefficients $b_{i}>0, i=0, \ldots, 6$ and

$$
\begin{gathered}
\Delta_{1}=\left|b_{1}\right|>0, \quad \Delta_{3}=\left|\begin{array}{lll}
b_{1} & b_{0} & 0 \\
b_{3} & b_{2} & b_{1} \\
b_{5} & b_{4} & b_{3}
\end{array}\right|>0, \\
\Delta_{5}=\left|\begin{array}{ccccc}
b_{1} & b_{0} & 0 & 0 & 0 \\
b_{3} & b_{2} & b_{1} & b_{0} & 0 \\
b_{5} & b_{4} & b_{3} & b_{2} & b_{1} \\
0 & b_{6} & b_{5} & b_{4} & b_{3} \\
0 & 0 & 0 & b_{6} & b_{5}
\end{array}\right|>0 .
\end{gathered}
$$

Therefore the square of the stability threshold $p_{\mathrm{FS} \text {,thr }}^{2}$ for the rotor-bearing system that considers the shaft flexibility is given by (Bently et al. [2]; Antonio-Garcia et al. [8])

$$
p_{\mathrm{FS}, \mathrm{thr}}^{2}=\frac{\Delta(c) \Delta(c, k) \Sigma^{2}(c)}{A+\Delta^{2}(c, k) \sum(c)+\Delta(k) \Sigma^{3}(c)-\Sigma(k) \Delta(c, k) \Sigma^{2}(c)},
$$

where

$$
A=\gamma\left[\Delta^{3}(c, k)+\Delta(c, k) \Delta(k) \Sigma^{2}(c)-\Delta^{2}(c, k) \Sigma(c) \Sigma(k)\right] .
$$

It is important to note that, when the shaft flexibility is set to zero $(\gamma=0)$, then $p_{\mathrm{FS} \text {, thr }}^{2}$ becomes $p_{\mathrm{RS}, \text { thr }}^{2}$, which corresponds to the stability threshold for rigid shafts.

Figure 5 shows the stability results for both cases. In Figure $5 \mathrm{a}$ the conventional circular bearing rotordynamics coefficients for the long bearing are used, and in Figure 5b the hybrid case with pressure supply $P_{s}=10$. Here, square of stability threshold values are plotted against the bearing equilibrium eccentricity for different shaft flexibility values $(\gamma)$, considering the previous rotordynamics coefficients. Notice that for $\gamma=0.1$ the improvement is dramatic, while for $\gamma=4$ the efficiency of the hybrid bearing is much diminished; yet the benefit that pressurized lubricant supply achieves can be highly appreciated for stabilization and control purposes.

Finally, the steady state imbalance responses of the Jeffcott rotor supported on conventional and hybrid bearings for different pressure supplies are shown in Figure 6. Both 


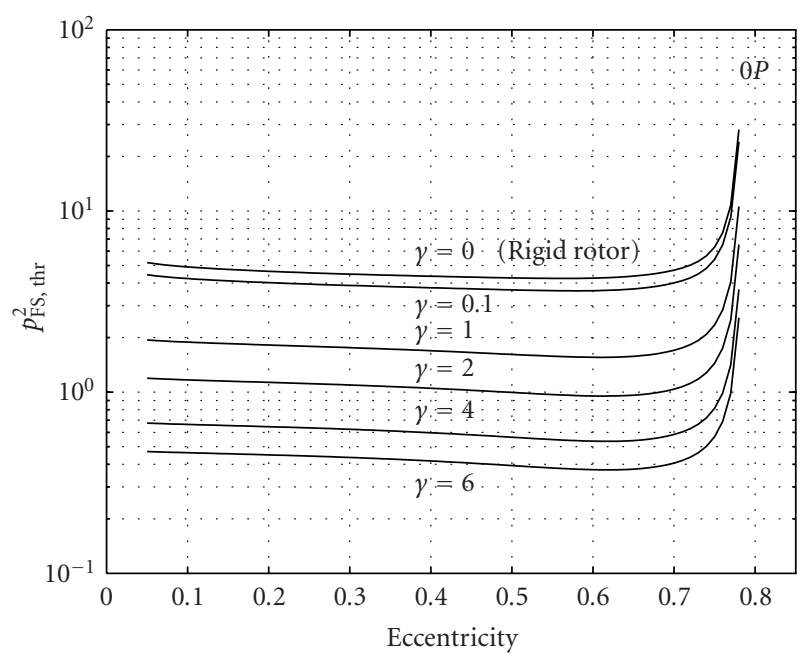

(a)

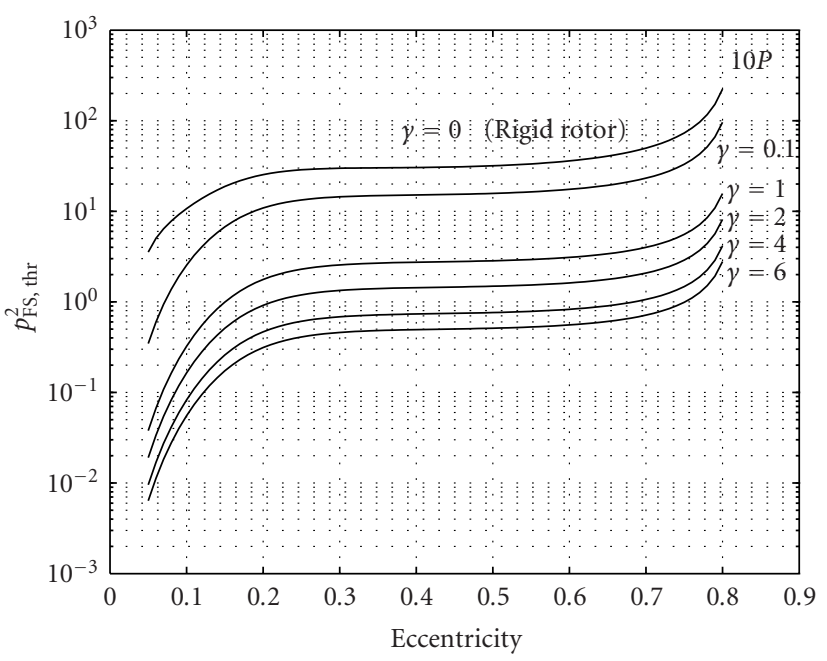

(b)

FIGURE 5: Stability threshold curves $p_{\text {FS,thr }}^{2}$ for different shaft flexibilities $\gamma$ : (a) conventional circular bearing $(0 P$, infinitely long bearing coefficients) and (b) hybrid, for pressure supply $P_{s}=10$.

system configurations are identical except in the disc mass where Figure $6 \mathrm{~b}$ has twice the mass as compared to Figure 6a. Observe that in both figures injecting lubricant while trespassing the critical speed is contra-productive but after this region, and only in case of Figure $6 a$, the pressurized injection can achieve reductions in the absolute disc amplitude.

\section{CONCLUSIONS}

Stability characteristics of conventional circular and hybrid bearing designs based on the Liénard-Chipart stability criterion, as far as equilibrium eccentricities and attitude angle are analyzed and compared. Rotordynamics coefficients

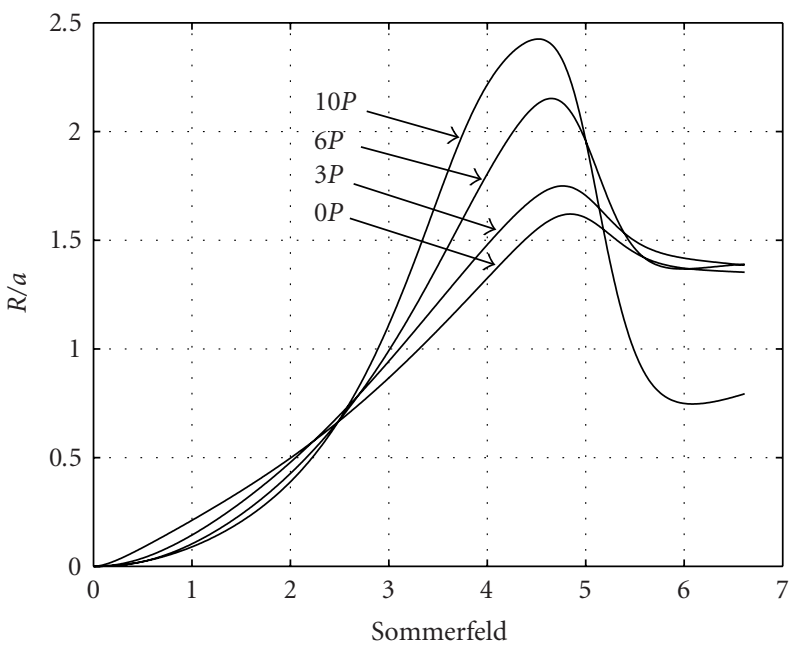

(a)

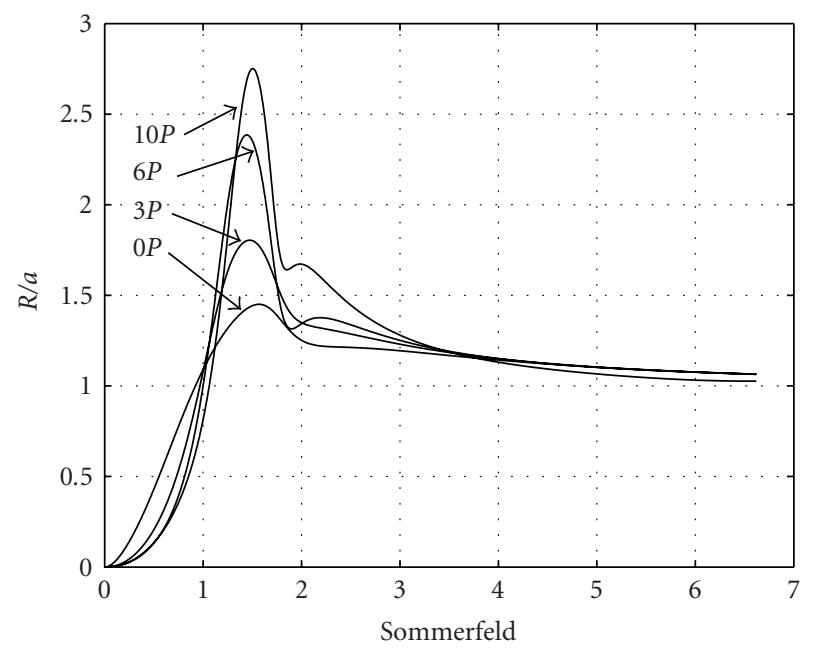

(b)

Figure 6: Steady state imbalance response of a Jeffcott rotor supported on hybrid bearing trespassing the critical speed: (a) semiflexible shaft and (b) flexible shaft with $\gamma$ equal to half of the case (a).

for the hybrid bearing design are numerically computed and data are curve-fitted and input into expressions to compare stability thresholds for both, conventional circular journal and hybrid bearing designs under diverse pressure supplies. The study comprises analysis of rigid rotors and flexible Jeffcott rotors supported on typical and hybrid bearings; thresholds curves for the conventional circular bearing type are much similar to those obtained by Lund [12]. Concerning the bearing equilibrium locus, the eccentricity increases and the attitude angle is reduced as the injection pressure increases.

Also study on the imbalance response of a simplified Jeffcott rotor supported on conventional and this hybrid bearing is performed; it is shown that the mass imbalance 
vibration can be effectively reduced (Ordoñez-Pantoja [6]). For this design, nonactive control and steady state vibration in Figure 6 leads to amplitude reduction capacities (at certain speed regions), where nonlinear behavior with respect to lubricant feed pressure is observed. Using the rotordynamics coefficients fitted curves into the stability expressions here derived, as well as the imbalance response performance of other type of bearings can be studied and evaluated.

Based on the previous expressions, in the analysis of the present design, and probably of other hybrid bearings (e.g., the Servofluid), proves that a proper pressurization design linked to an adequate vibration control system can be applied to improve rotating machine performances supported on these components.

\section{ACKNOWLEDGMENTS}

This work was partially sponsored by Consejo Nacional de Ciencia y Tecnologia (CONACyT), Mexico, through research project 38711-U. Also thanks to the SNI and the EDI scholarships, granted by CONACyT and Instituto Politecnico $\mathrm{Na}$ cional de Mexico, respectively.

\section{REFERENCES}

[1] D. Bently, J. Grant, and P. Hanifan, "Active-control fluid bearings for a new generation of machines," Orbit, vol. 20, no. 2, 1999.

[2] D. Bently, T. Elridge, and J. Jensen, "Externally pressurized bearings allow rotor dynamic optimization," in Proc. International Symposium on Stability and Control of Rotating Machinery (ISCORMA '01), p. paper 3018, Lake Tahoe, Calif, USA, August 2001.

[3] V. V. Kucherenko and J. C. Gomez-Mancilla, "Bifurcations of an exactly solvable model of rotordynamics," Internat. J. Bifur. Chaos Appl. Sci. Engrg., vol. 10, no. 12, pp. 2689-2699, 2000.

[4] J. Gomez-Mancilla, "Technological developments in rotordynamics," Project and final report, Feria Internacional El Salvador, Mexico, DF, Mexico, 1997.

[5] F. Dimofte and R. Hendricks, "Active controlled fluid film based on wave bearing technology," in Proc. International Symposium on Stability and Control of Rotating Machinery (ISCORMA '01), p. paper 3014, Lake Tahoe, Calif, USA, August 2001.

[6] A. Ordoñez-Pantoja, "Design and analysis of a controllable hybrid bearing," M.S. thesis, Escuela Superior de Ingeniería Mecánica y Eléctrica (ESIME), Instituto Politecnico Nacional, Mexico, DF, Mexico, 2003.

[7] G. Meng and R. Gash, "Stability and stability degree of a cracked flexible rotor supported on journal bearings," ASME Trans. Journal of Vibration and Acoustics, vol. 122, no. 2, pp. 116-125, 2000.

[8] A. Antonio-Garcia, J. Gomez-Mancilla, and V. Nosov, "Stability threshold speed calculation for rigid and flexible jeffcott rotors," in Proc. 3er Congreso Nacional de Ingenieria Electromecanica y de Sistemas (ICESE '02), IPN, Mexico, DF, Mexico, November 2002.

[9] V. N. Afanasiev, V. B. Kolmanovskii, and V. R. Nosov, Mathematical Theory of Control System Design, Kluwer Academic Publishers, Boston, Mass, USA, 1996.

[10] B. P. Demidovich, Lectures on the Mathematical Theory of Stability, Nauka Press, Moscow, Russian, 1967.
[11] J. Gomez-Mancilla and A. D. Dimarogonas, "MAQUI/ CHUMA/FFT," Clave SEP-86441, CopyRight, Mexico, Df, Mexico, 1996.

[12] J. W. Lund, Self-Excited, Stationary Whirl Orbits of a Journal in a Sleeve Bearing, Ph.d. dissertation, Rensselaer Polytechnic Institute, Troy, NY, USA, 1966. 

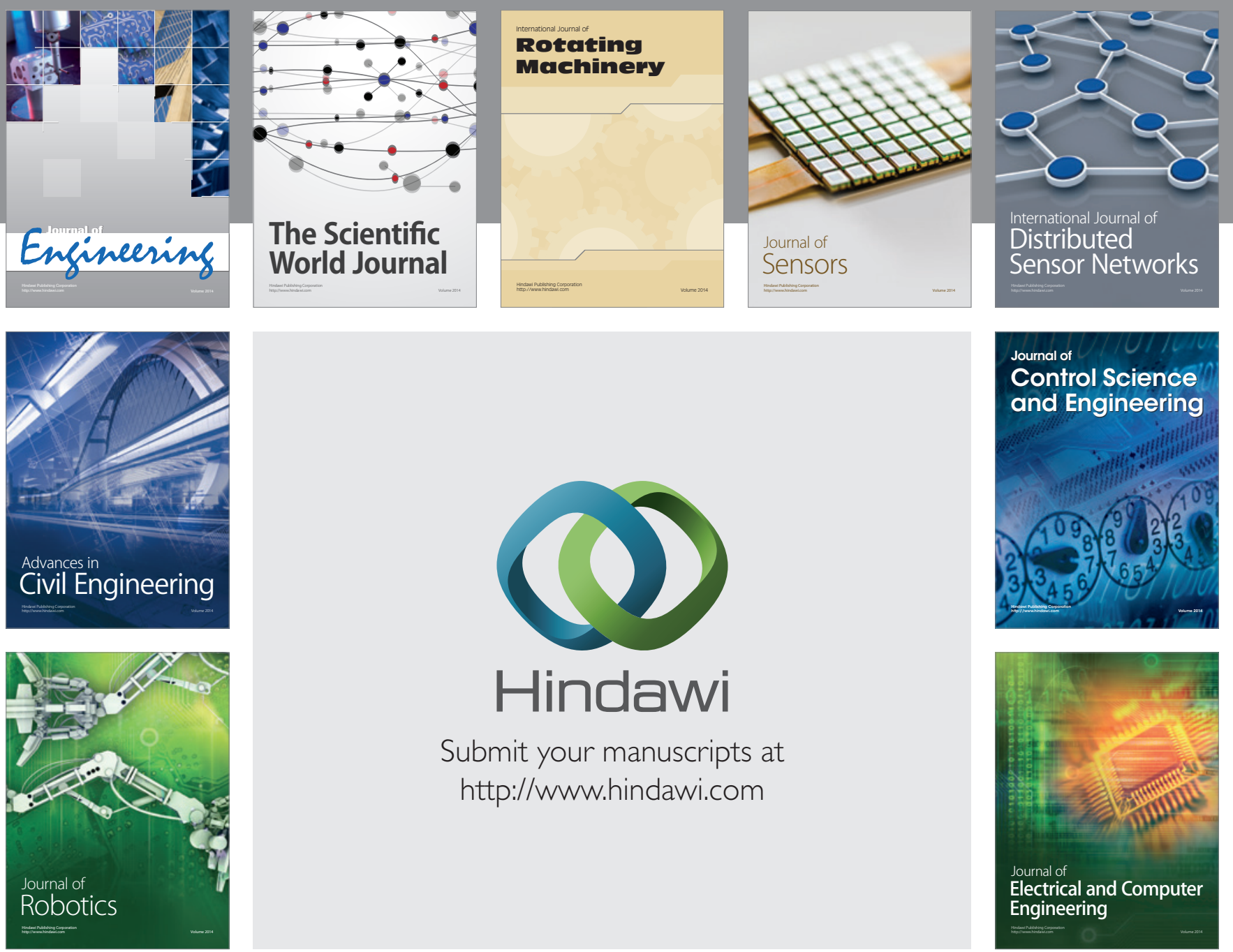

Submit your manuscripts at

http://www.hindawi.com
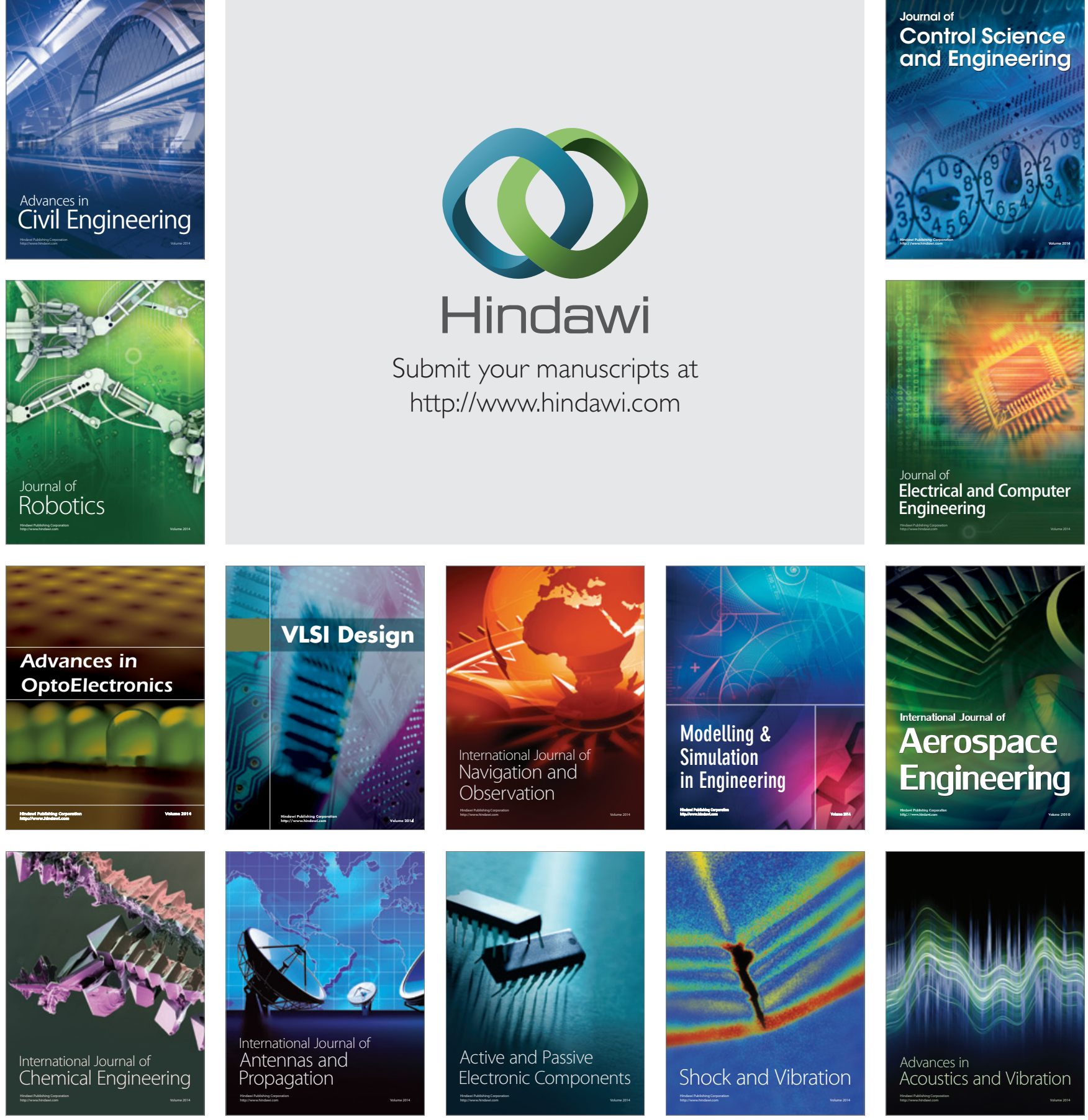